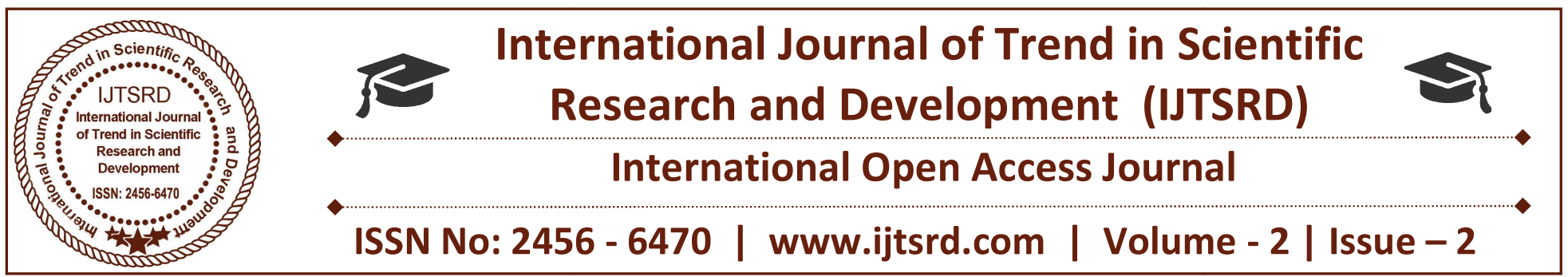

\title{
Analog CMOS Image Sensor-based Radon Counter
}

\author{
Chungyong Kim \\ Department of Electrical and Computer Eng., \\ University of Seoul, Seoul, Korea
}

\author{
Gyu-Sik Kim \\ Department of Electrical and Computer Eng., \\ University of Seoul, Seoul, Korea
}

\begin{abstract}
Radon is an invisible, odorless, and chemically inactive radioactive gas produced by the decay of uranium ore. Various types of equipment and components have been proposed for use in effective radon detection. In this paper, we describe a radon detector that uses an analog CMOS image sensor module. Based on our studies, we believe that this system would be helpful in protecting many people from the dangers associated with radon exposure.
\end{abstract}

Keywords: radon, radon detection, CMOS image sensor module, radon exposure

\section{Introduction}

Radon is a natural, inert, invisible, odorless, chemically inactive, and radioactive gas emitted by the earth. Because it is inert and does not chemically bond to elements, it is released from soil into the atmosphere. Because inhaling radon and its radioactive-decay products causes irradiation of lung tissue, prolonged exposure to a high concentration of radon significantly increases the risk of developing cancer.

There are many commercial instruments and techniques available for measuring radon indoors. In [1], a high-sensitivity radon detector that uses an electrostatic collection and a PIN photodiode for air and water was developed. A calibration system for the humidity-dependence measurement has also been developed. In [2], The PIN photodiode radon detector was improved for the highly sensitive measurement of low-level radon concentrations. The system was shown to have a sensitivity to radon concentrations as low as $1.6 \mathrm{mBq} / \mathrm{m}^{3}$. In [3], a super high-sensitivity radon detector for water was developed. This detector is used as a real-time monitor of radon concentration in water at the Super-Kamiokande neutrino observatory in Japan. In [4], they showed that silicon PIN photodiodes could be successfully used for thermal neutron measurements with ${ }^{6} \mathrm{LiF}$ converters and tritons created in the converter were very well detected by the diode. In [5], they developed a lowcost continuous radon measurement system using a silicon PIN photodiode. It could be applied to laboratory and field determinations, and to measure accurately radon emanation rates from porous materials. In [6], a radon in air detecting device using a silicon PIN photodiode was developed and studied. They found that an inverse bias voltage of $-9 \mathrm{~V}$ was good choice for measuring. In [7], they assessed the performance of the Complementary Metal-Oxide Semiconductor (CMOS) electronic system for $\alpha$ particle detection from sources and in a ${ }^{222} \mathrm{Rn}$ atmosphere and compared the experimental results with those obtained from conventional methods. In [8], an integrated system-on-chip (SoC) has been designed in $0.6 \mu \mathrm{m}$ CMOS mixed analog/digital technology, and tested for high rate alpha particle counting. In [9], they presented the complete electronic board of the future electronic radon monitor (ERM): it was made of three independent AlphaRad chips running in parallel, mounted on a small printedcircuit board which included a numeric block for data treatment based on a Xilinx programmable gate array. In [10], they described a fully standalone compact electronic monitor for atmospheric ${ }^{222} \mathrm{Rn}$, based on a new CMOS SoC. In [11], a passive direct-reading 
radon monitor utilizing a custom $\alpha$ particle detecting MOS integrated circuit and electrostatic radon progeny concentrator has been designed. In [12], a silicon PIN photodiode was designed and fabricated in consideration of low-leakage-current and high-biasvoltage application. In [13], a fast-responding passive radon detector using electrostatic concentration and enhanced readout electronics has been designed. In [14], the system is developed which monitors the radon level, using a PIN diode for detecting the radon particles and a data processing module with Wi-Fi communication capabilities for the transmission and management of measurement results.

For our experiments, we implemented a radon counter using a CMOS image sensors module. These radon counters were used to measure the radon concentration of indoor air in houses and workplaces.

\section{Implementation of Radon Counters}

\subsection{CCD and CMOS image sensors:}

An image sensor is a sensor that detects and conveys the information that constitutes an image. It does so by converting the variable attenuation of light waves (as they pass through or reflect off objects) into signals, small bursts of current that convey the information. The waves can be light or other electromagnetic radiation. Image sensors are used in electronic imaging devices of both analog and digital types, which include digital cameras, camera modules, medical imaging equipment, night vision equipment such as thermal imaging devices, radar, sonar, and others. When an image is captured by a network camera, the light passes through the lens and falls on the image sensor. The image sensor consists of picture elements, also called pixels, which register the amount of light that falls on them. They convert the received amount of light into a corresponding number of electrons. The stronger the light, the more electrons are generated. The electrons are converted into voltage and then transformed into numbers by means of an A/D-converter. The signal constituted by the numbers is processed by electronic circuits inside the camera.

Presently, there are two main technologies that can be used for the image sensor in a camera, i.e. CCD (Charge-coupled Device) and CMOS (Complementary Metal-oxide Semiconductor). CCD image sensors accomplish task of capturing light and converting it into electrical signals. Each cell of a CCD image sensor is an analog device. When light strikes the chip, it is held as a small electrical charge in each photo sensor. The charges are converted to voltage signal for one pixel at a time as they are read from the chip. This signal is amplified outside the sensor (Figure 1). CCD sensors have had some advantages compared to CMOS sensors, such as better light sensitivity and less noise. In recent years, however, these differences have disappeared. The disadvantages of CCD sensors are that they are analog components that require more electronic circuitry outside the sensor. CCD sensors also require a higher data rate, since everything has to go through just one output amplifier, or a few output amplifiers.

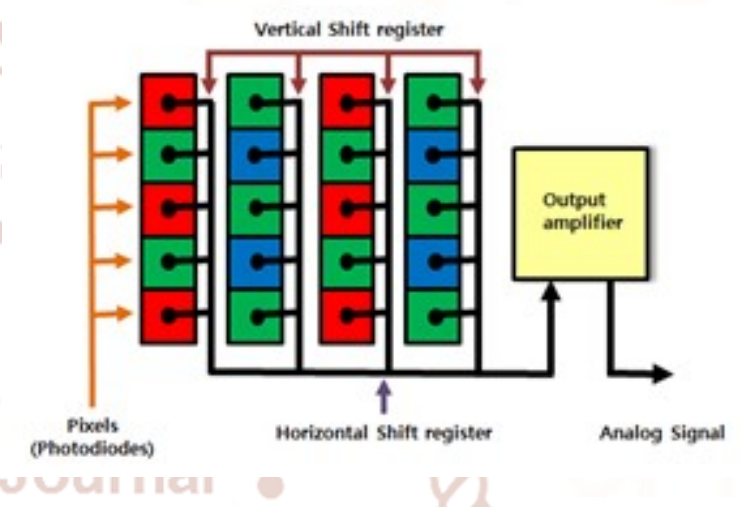

\section{Fig 1: CCD operation principle}

Ordinary CMOS chips were used for imaging purposes, but the image quality was poor due to their inferior light sensitivity. Modern CMOS sensors use a more specialized technology and the quality and light sensitivity of the sensors have rapidly been increased in recent years. CMOS chips have several advantages. Unlike the CCD sensor, the CMOS chip incorporates amplifiers and A/D-converters, which lowers the cost for cameras since it contains all the logics needed to produce an image (Figure 2). Every CMOS pixel contains conversion electronics. Compared to CCD sensors, CMOS sensors have better integration possibilities and more functions. However, this addition of circuitry inside the chip can lead to a risk of more structured noise, such as stripes and other patterns. CMOS sensors also have a faster readout, lower power consumption, higher noise immunity, and a smaller system size. Our radon counter system measures radon gas concentration using CMOS image sensor in camera at very low cost. Alpha particles emitted by alpha decay of radon are collected by an electrostatic concentrator, and strike one or more pixels of CMOS image sensor and they are counted. The concentrator which is applied by high voltage generates sufficient charge to saturate pixels. 


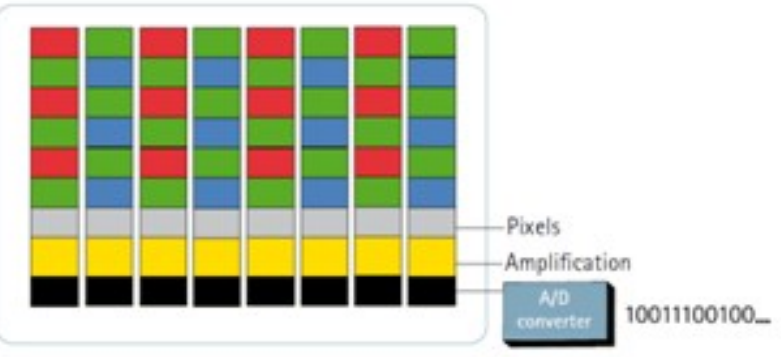

Fig 2: CMOS operation principle

\subsection{Arduino MCU and LCD display modules:}

The Mega 2560 is a microcontroller board based on the ATmega2560. It has 54 digital input/output pins (of which 15 can be used as PWM outputs), 16 analog inputs, 4 UARTs (hardware serial ports), a $16 \mathrm{MHz}$ crystal oscillator, a USB connection, a power jack, an ICSP header, and a reset button. It contains everything needed to support the microcontroller; simply connect it to a computer with a USB cable or power it with an AC-to-DC adapter or battery to get started. The Mega 2560 board is compatible with most shields designed for the Uno and the former boards Duemilanove or Diecimila.

LCD (Liquid Crystal Display) screen is an electronic display module and find a wide range of applications. A $16 \times 2$ LCD display is very basic module and is very commonly used in various devices and circuits. These modules are preferred over seven segments and other multi segment LEDs. The reasons being: LCDs are economical; easily programmable; have no limitation of displaying special \& even custom characters (unlike in seven segments), animations and so on. A $16 \times 2$ LCD means it can display 16 characters per line and there are 2 such lines. In this LCD each character is displayed in $5 \times 7$ pixel matrix. This LCD has two registers, namely, Command and Data. The command register stores the command instructions given to the LCD. A command is an instruction given to LCD to do a predefined task like initializing it, clearing its screen, setting the cursor position, controlling display etc. The data register stores the data to be displayed on the LCD. The data is the ASCII value of the character to be displayed on the LCD.

The LCD display chosen in this paper is a LCD module (BC4915AYPLEH) that shows the level of radon gas in Pico Curies per liter $(\mathrm{pCi} / \mathrm{L})$. The display range is 0.0 to 999.9 . The radon counter developed in this paper is designed to notify the user of the level of radon gas on either a short-term or long-term basis, and is updated every hour if there is a change in the level of radon gas.

\subsection{Radon detection using an analog CMOS sensor module:}

In [15], a webcam CMOS image sensor is used to detect the alpha emissions from radon progeny, in particular, 218Po and 214Po which have been concentrated onto the image sensor surface (Fig. 3). The cover glass of a CMOS image sensor module should be removed in order to make the alpha emissions hit the image sensor surface. This type of radon detector can be implemented at low cost. But, it needs a very powerful processor such as a personal computer (PC) in order to analyze the webcam data of big size. So, for a portable low-end real time radon counter, we chose an analog CMOS image sensor. Figure 4 shows the analog CMOS image sensor module used for our experimental studies.

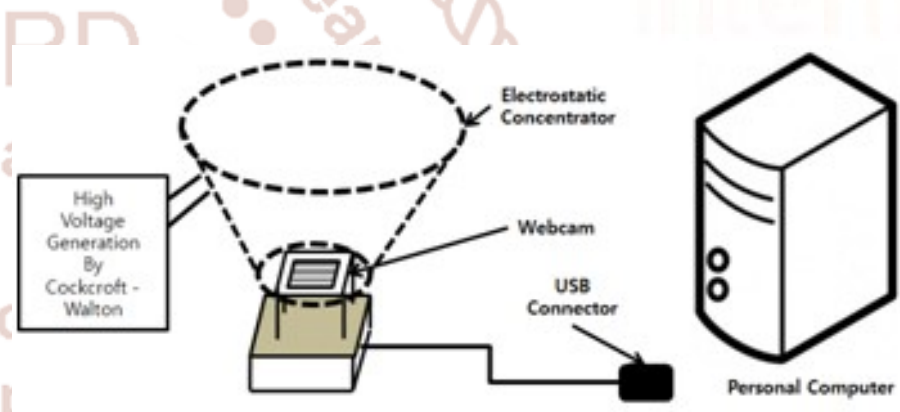

Fig 3: Radon counter using a webcam CMOS image sensor

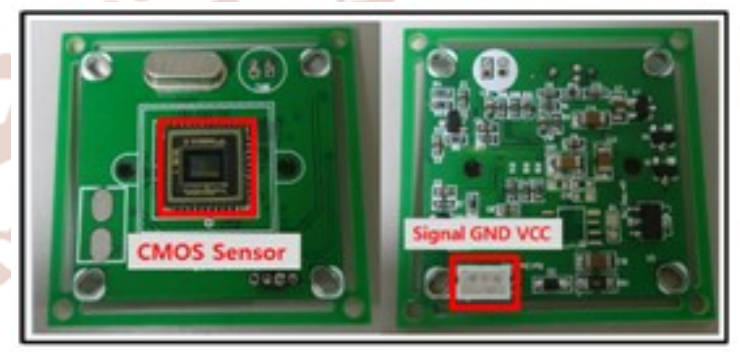

Fig 4: Analog CMOS image sensor module (RY1030) used for experimental studies

Fig. 5 is presented in order to compare these two methods - digital CMOS radon counter and analog CMOS radon counter. In Fig. 5 (a), webcam control and analysis routines are written in MATLAB using the image acquisition and image processing toolboxes. Data are read in 50 frames at a time and then analyzed. Therefore, it needs a very powerful processor such as a PC in order to analyze the webcam data of big size. On the other hand, the radon 
signal can be easily detected by using a simple comparator circuit because the output of an analog CMOS image sensor is an analog signal as shown in Fig. 5 (b).

In order to test the radon counting method using an analog CMOS image sensor module, an analog CMOS image sensor module was placed in a lightblocking chamber as can be seen in Fig. 6. Normally, the output voltage of the CMOS image sensor signal was under $3.3 \mathrm{~V}$. But, when the radon particle was detected, it was over $3.7 \mathrm{~V}$ as shown in Fig. 6 (b). Therefore, the comparator circuit with reference voltage of $3.5 \mathrm{~V}$ can be used for the radon detection.

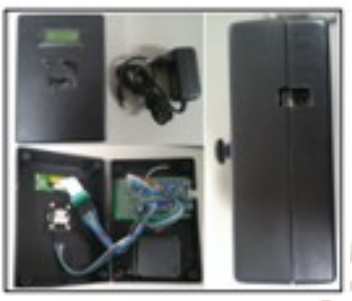

(a)

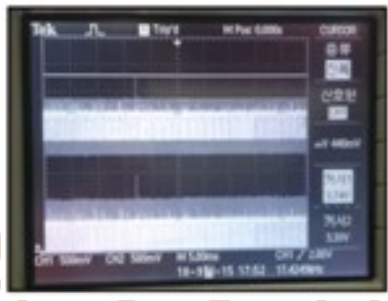

(b)

Fig 6: Analog CMOS image sensor module in a light-blocking chamber (a) and detected radon (b)

The circuit design of power, PWM, LCD, switch, LED, buzzer, MCU, and sensor circuits is made. Based on the circuit design, the CMOS radon counter PCB layout is made as shown in Fig. 7 (a). Finally, the analog CMOS radon counter is implemented as Fig. 7 (b).

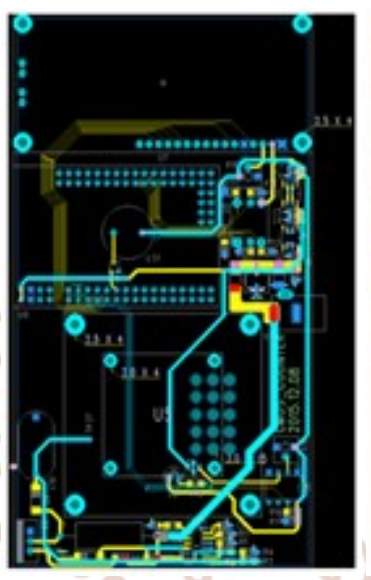

(a)

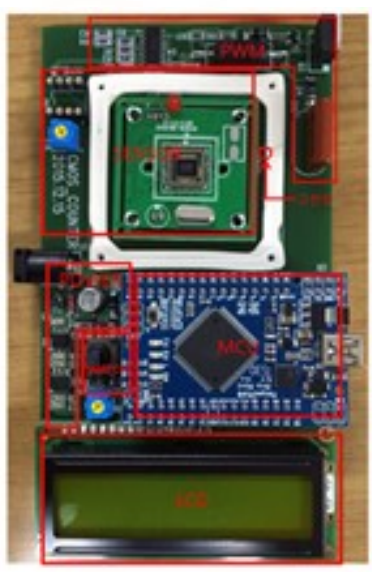

(b)
Fig 7: PCB layout (a) and assembled CMOS radon counter (b)

\section{Experimental Results and Discussion}
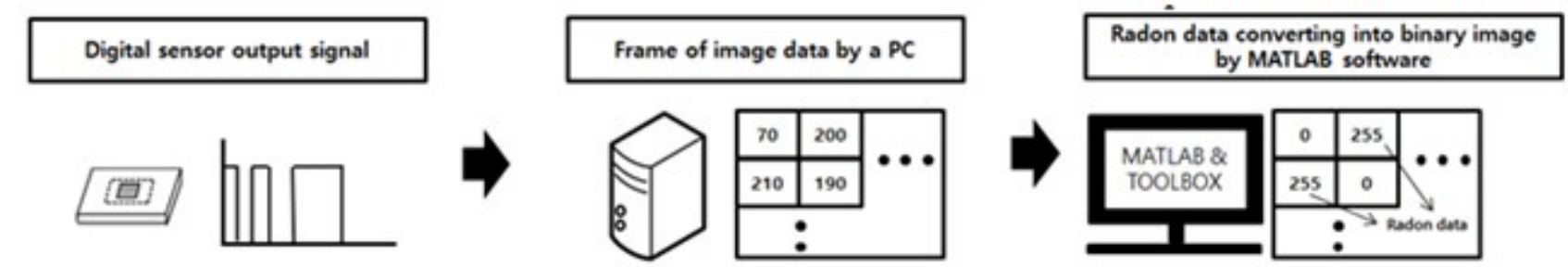

(a)
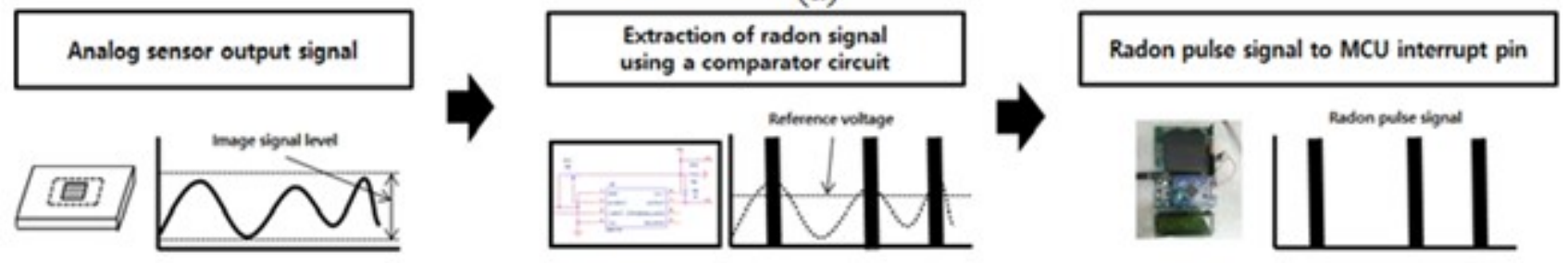

(b)

Fig 5: Radon counting using a digital CMOS image sensor (a) and an analog CMOS image sensor (b)

The RAD7 radon detector, manufactured by Durridge Company in the United States, is a highly versatile instrument that forms the basis of a comprehensive radon measurement system. It is used in many different modes for different purposes.
The analog CMOS radon counter implemented in our tests was calibrated using the RAD7 electronic radon detector shown in Fig. 8. The RAD7 uses an air pump and a solid-state alpha detector that is made of semiconductor material that converts alpha radiation directly into an electrical signal. It has desiccant $\left(\mathrm{CaSO}_{4}\right)$ tubes and inlet filters (pore size $\left.1 \mu \mathrm{m}\right)$ that 
block fine dust particles and radon daughters from entering the radon test chamber. The RAD7's internal sample cell is a 0.7 -liter hemisphere coated on the inside with an electrical conductor. A silicon alpha detector occupies the center of the hemisphere.

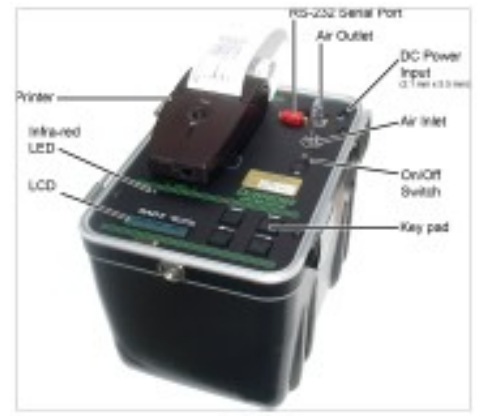

Fig 8: RAD7 electronic radon detector

The methyl methacrylate box made for radon concentration calibration is shown in Fig. 9. The calibration experiments were conducted for $154 \mathrm{~h}$ using a varied concentration of radon gas. Using a linear regression-analysis technique, the radon counts per hour of the implemented analog CMOS radon counters were calibrated to $\mathrm{pCi} / \mathrm{L}$ which is a unit of radon concentration. Fig. 10 shows the linear regression analysis for 12 hours data average. Fig. 11 shows the case of 24 hours data average. These experiment results suggest that the implemented analog CMOS radon counters could prove useful for measuring and monitoring radon concentration of the indoor air in a house or a workplace.

\section{Concluding Remarks}

Because inhaling radon and its radioactive-decay products causes irradiation of lung tissue, prolonged exposure to a high concentration of radon significantly increases the risk of developing cancer.

In this paper, a radon counter using a CMOS radonsensor module was discussed. This radon counter can be used to measure radon concentration of the indoor air in houses and workplaces. The data collected by the radon counters in houses and workplaces was transferred using $\mathrm{Wi}-\mathrm{Fi}$ and the Internet to a radonmonitoring database created on a server, which stored client data. If this radon remote-sensing Wi-Fi-based monitoring system is used in army barracks, classrooms, and country houses where there is a risk of high levels of radon exposure, soldiers, students, and residents could be protected from the dangers of radon exposure.

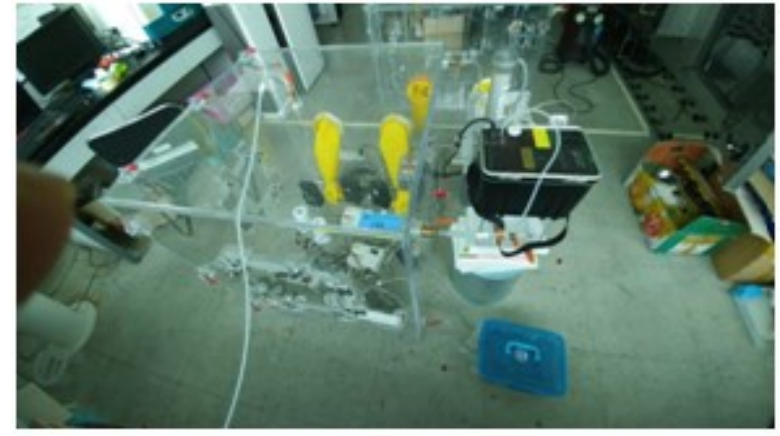

Fig 9. Methyl methacrylate box made for radon concentration calibration

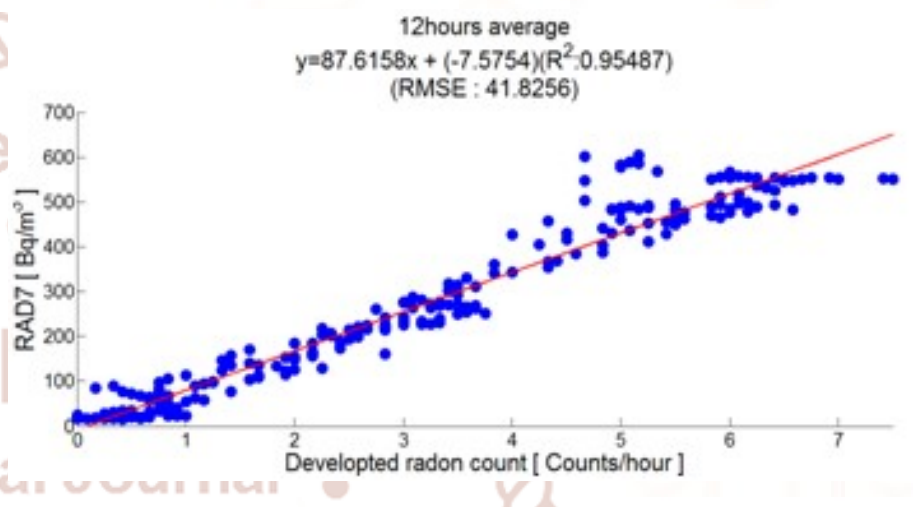

Fig 10: Linear regression analysis for 12 hour averaged radon data
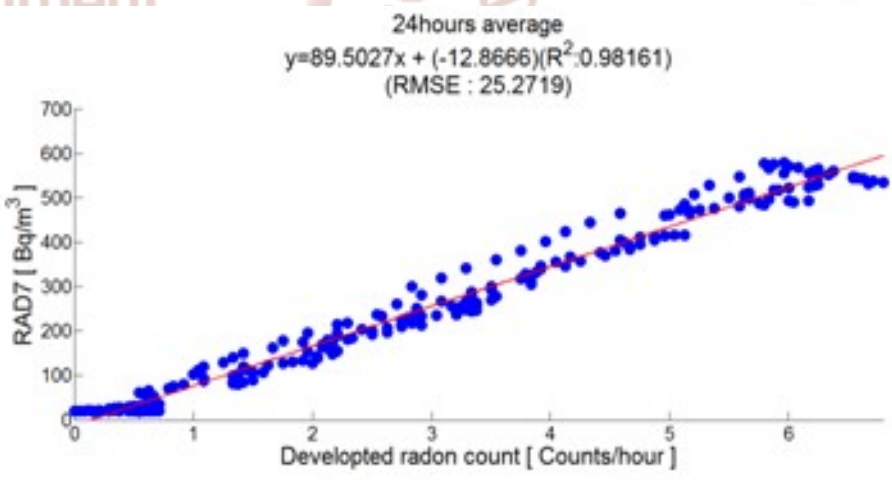

Fig 11: Linear regression analysis for 24 hour averaged radon data

\section{Acknowledgment}

This work was also supported by the National Research Foundation of Korea (NRF) grant funded by the Korea government (MSIP) (No. 2016011249). 


\section{REFERENCES}

1. Y. Takeuchi, K. Okumura, T. Kajita, S. Tasaka, H. Hori, M. Nemoto, and H. Okazawa, "Development of high sensitivity radon detectors," Nuclear Instruments \& Methods in Physics Research A 421, pp.334-341, 1999

2. E. Choi, et al., "Highly sensitive radon monitor and radon emanation rates for detector components," Nuclear Instruments \& Methods in Physics Research A 459, pp.177-181, 2001

3. C. Mitsuda, T. Kajita, K. Miyano, S. Moriyama, M. Nakahata, Y. Takeuchi, S. Tasaka, "Development of super-high sensitivity radon detector for the Super-Kamiokande detector," Nuclear Instruments \& Methods in Physics Research A 497, pp.414-428, 2003

4. M. Voytchev, et al., "Neutron detection with a silicon PIN photodiode and "LiF converter," Nuclear Instruments \& Methods in Physics Research A 512, pp.546-552, 2003

5. J.L. Gutiérrez, et al,, "Radon emanation measurements using silicon photodiode detectors," Applied Radiation and Isotopes, Vol.60, pp.583-587, 2004

6. A. Martín-Martín, et al., "Radon measurements with a PIN photodiode," Applied Radiation and Isotopes, Vol.64, pp.1287-1290, 2006

7. A. Nourreddine, A. Nachab, D. Husson, and S. Higueret, "MCNPX simulation for $\alpha$-particle detection by CMOS active pixel sensor," Radiation Measurements, Vol.40, pp.275-278, 2005

8. D. Husson, A. Bozier, S. Higueret, T.D. Le, and A. Nourreddine, "AlphaRad, a new integrated CMOS System-on-Chip for high eficiency alpha particles counting," Nuclear Instruments \&
Methods in Physics Research A 569, pp.845-852, 2006

9. S. Higueret, D. Husson, T.D. Le, and A. Nourreddine, and N. Michielsen, "Electronic radon monitoring with the CMOS System-onChip AlphaRad," Nuclear Instruments \& Methods in Physics Research A 584, pp.412-417, 2008

10. S. Higueret, D. Husson, M. Tromcme, A. Nourreddine, T.D. Le, and N. Michielsen, "Measurement of ${ }^{222} \mathrm{Rn}$ at the $\mathrm{Bqm}^{-3}$ level with the AlphaRad chip," Radiation Measurements, Vol.43, pp.1059-1062, 2008

11. R. H. Griffin, H. Le, D.T. Jack, A. Kochermin, and N.G. Tarr, "Radon monitor using custom $\alpha$ detecting MOS IC," in Proceedings of IEEE Sensors 2008, pp.906-909, Oct. 2008

12. Han Soo Kim, Se Hwan Park, Jang Ho-Ha, DongHoon Lee, and Seung Yeon Cho, "Characteristics of a fabricated PIN photodiode for a matching with a CsI(TI) scintillator," IEEE Trans. Nucl. Sci., Vol.57, No.3, pp. 1382-1385, June, 2010

13. R.H. Griffin, A. Kochermin, N.G. Tarr, H.McIntosh, H.Ding, J.Weber and R.Falcomer, "A sensitive, fast-responding passive electrostatic radon monitor," in Proceedings of IEEE Sensors 2011, Oct., 2011

14. S. Folea, M. Hulea, G.Mois, V. Cosma, "Wi-Fi portable solution for distributed radon measurements," Rom. Journ. Phys., Vol.58, pp.S126-s139, 2013

15. Ryan H. Griffin and N. Garry Tarr, "Optical image sensors and their application in radon detection," in Proceedings of SPIE, Vol.8915, pp.8915C-1 7, Oct., 2013 